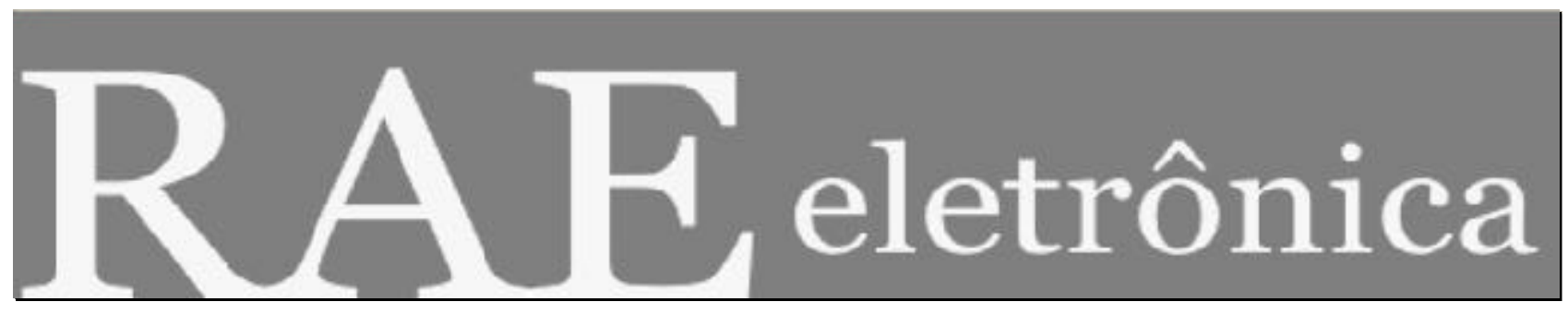

\title{
DETERMINANTES DA DISSEMINAÇÃO VOLUNTÁRIA DE INFORMAÇÕES FINANCEIRAS NA INTERNET
}

Por:

\author{
Wesley Mendes -da-Silva, FIR \\ Paulo Azevedo de Oliveira Magalhães Filho, FIR
}

RAE-eletrônica, v. 4, n. 2, Art. 19, jul./dez. 2005

http://www.rae.com.br/eletronica/index.cfm?FuseAction=Artigo \&ID=2388\&Secao=ARTIGOS\&Volu me $=4 \&$ Numero $=2 \&$ Ano $=2005$

CCopyright, 2005, RAE-eletrônica. Todos os direitos, inclusive de tradução, são reservados. É permitido citar parte de artigos sem autorização prévia desde que seja identificada a fonte. A reprodução total de artigos é proibida. Os artigos só devem ser usados para uso pessoal e nãocomercial. Em caso de dúvidas, consulte a redação: raeredacao@fgvsp.br.

A RAE-eletrônica é a revista on-line da FGV-EAESP, totalmente aberta e criada com o objetivo de agilizar a veiculação de trabalhos inéditos. Lançada em janeiro de 2002, com perfil acadêmico, é dedicada a professores, pesquisadores e estudantes. Para mais informações consulte o site www.rae.com.br/eletronica.

\section{RAE-eletrônica}

ISSN 1676-5648

(C2005 Fundação Getulio Vargas - Escola de Administração

de Empresas de São Paulo.

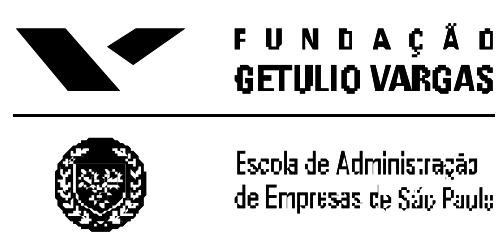




\title{
RESUMO
}

A transparência com que as empresas disponibilizam informações ao mercado tem sido reconhecida como um dos principais pilares das boas práticas de governança. Paralelamente, o antigo paradigma da comunicação passa por transformações decorrentes da era digital. As empresas estão aumentando a utilização da Internet como meio de relacionamento com os investidores, e com isso estão oferecendo maiores graus de transparência ao mercado. Este artigo tem como objetivo verificar a existência de associações entre características das empresas e a disseminação voluntária de informações financeiras usando a rede mundial de computadores, tomando como referência as empresas brasileiras. $\mathrm{O}$ estudo consiste em um cross section realizado a partir de dados referentes a 291 empresas não-financeiras listadas na Bovespa em 2002. As evidências sugerem que um maior tamanho da empresa, assim como um menor retorno anual de suas ações, está associado a uma maior soma de informações financeiras disseminadas no site corporativo.

\begin{abstract}
The transparency with which companies disclose information to the market has been recognized as one of the main pillars of the good practices of Corporate Governance. In addition, the old paradigm of the communication passes through transformations deriving from the Digital Age. Therefore, companies are increasingly using the Internet as a means of investor relations, consequently offering a higher degree of disclosure to the market. The aim of this sarticle aims is to verify the existence of a relationship between characteristics of the companies and the voluntary disclosure of financial information through the World Wide Web, taking Brazilian companies as reference. The study consists in a cross section of data from 291 non-financial companies listed in the São Paulo Stock Exchange in 2002. The evidence suggests that company size as well as smaller annual stock returns are associated to greater amounts of financial information in the corporate Website.
\end{abstract}

\section{PALAVRAS-CHAVE}

Governança corporativa, disclosure, Internet, empresas brasileiras, finanças corporativas.

\section{KEY WORDS}

Corporate governance, Disclosure, Internet, Brazilian firms, Corporate finance. 


\section{INTRODUÇÃO}

A sociedade vem impondo padrões de comportamento às empresas, com respeito à forma de tratamento dos acionistas e do ambiente em geral. Isso ocorre simultaneamente com a mudança do paradigma da comunicação. $\mathrm{O}$ advento da Internet proporcionou às corporações um inovador e poderoso canal de relacionamento com investidores. Assim, cada vez mais as grandes companhias abertas estão utilizando a Web para uma aproximação maior com seus acionistas, analistas e investidores. Ao democratizar o acesso às informações, a empresa está preservando um dos requisitos da boa governança corporativa, a transparência, além de colaborar para a eqüidade no tratamento dos proprietários.

Na óptica de Zingales (2000), a influência da mídia não deveria ser negligenciada nos estudos desenvolvidos em Economia e Finanças. De maneira complementar, autores como Dyck e Zingales (2002) argumentam que o processo de difusão das informações (disclosure) ainda possui pouca representatividade em modelos econômicos. No entanto, um número cada vez maior de pessoas busca informações na mídia, notadamente na rede mundial de computadores.

Nesse contexto, a Internet tem aumentado consideravelmente sua atuação como ferramenta de trabalho para investidores. Isto é, em países de sistemas econômicos mais desenvolvidos, as companhias utilizam suas páginas na Web como plataforma para disponibilizar informações financeiras e relatórios úteis ao mercado e aos stakeholders (Ismail, 2000). No âmbito internacional, algumas pesquisas já abordaram as associações entre características corporativas, tais como tamanho, alavancagem e rentabilidade, com sua voluntariedade em disponibilizar informações financeiras ao mercado pela Internet, destacando-se Brennan e Hourigan (2000) e Ettredge, Richardson e Scholz (2002). Mas a falta de padronização do uso da Internet por parte das empresas tem possibilitado uma variedade de formas de sua exploração para comunicação com o mercado.

O objetivo deste artigo é verificar a existência de associações entre características das empresas, quais sejam: alavancagem, retorno anual das ações, tamanho e rentabilidade, com a disseminação de informações financeiras por meio da Internet. O estudo desenvolve-se com dados referentes a 291 empresas não-financeiras listadas na Bovespa no ano de 2002. Espera-se que os resultados deste trabalho auxiliem, orientem e estimulem a adoção de políticas de utilização da Internet como meio de difusão voluntária de relatórios financeiros no âmbito nacional. Os principais resultados obtidos sugerem a existência de associações positivas e significativas entre o tamanho da empresa e as proxies de disseminação voluntária de informações financeiras e ainda o fato de que as empresas que exibiram menores retornos anuais de suas ações divulgaram uma parcela maior de informações financeiras.

Esta pesquisa está organizada em cinco partes. A segunda seção apresenta a literatura relevante acerca da temática. A seguir, é apresentada a metodologia adotada para a realização da pesquisa. Logo após, são apresentados e discutidos os resultados empíricos obtidos no estudo e, na quinta seção, desenvolvem-se os argumentos da conclusão. 


\title{
BASES TEÓRICAS DO ESTUDO
}

Assim como argumentam La Porta et al. (1998), na América Latina, parecem ainda ser incipientes a discussão e a elaboração de políticas de gerenciamento com base em princípios de governança corporativa, apesar de já existirem iniciativas de diversos setores da sociedade com vistas às suas difusão e valorização no âmbito das economias emergentes. Adicionalmente, à luz da evolução da tecnologia da informação, já se verifica na literatura acerca do tema debatido neste artigo a preocupação com a adoção de novas formas de disseminação de informações:

\begin{abstract}
Nowadays, the most extended communication channel for financial information disclosure in local entities consists in a printed copy of the budget and the annual financial reports deposited in the entity. Even though this copy is kept for public consultation, applicants are frequently not authorised. While most local managers admit that any person that is interested may examine financial statements, various circumstances such as the lack of habit or work overload are behind their unwillingness to grant access. This difficulty to access public information considerably limits research possibilities in this field, especially when trying to gather panel data on a large number of entities. The high costs of data gathering are other important limitations of the current situation in most entities, even when access is granted. Today, the search for enhanced accessibility makes unavoidable the use of the new information technologies, namely the Internet. The use of the new information technologies has an enormous impact on the standards of availability and diffusion of information, introducing determinant advantages as readiness, low effort, and low cost in communication (Isenmann e Lenz, 2001, p. 169-78).
\end{abstract}

Embora já exista ao redor do mundo uma quantidade razoável de estudos relacionados aos fatores determinantes da disseminação voluntária de informações financeiras pela Internet, no âmbito nacional essa questão ainda é pouco explorada. Porém, em mercados mais desenvolvidos que o brasileiro, já se nota preocupação com esse aspecto:

The demand- and supply-side implications of the Internet for corporate disclosure are profound. If companies can use the Net to access information almost constantly and instantaneously - and investors and analysts have a thirst for such information - the obvious result will be much more continuous reporting of financial and business information. Much, if not all, of this information should be designed to help investors better estimate companies' future profitability and relative riskiness so that they can more accurately price companies' true market values (Litan e Wallison, 2000, p. 8).

Entretanto, há problemas associados ao uso da Internet nela quase inexiste padronização. Em alguns países já há algumas tentativas de inserir orientações para a disseminação de informações financeiras pela Web, sendo contemplados essencialmente: autenticidade e credibilidade das informações financeiras e ainda os relatórios divulgados pelos auditores (Debreceny e Gray, 1999); 
segurança e coerência entre relatórios financeiros estatutários e outras informações financeiras online (Koreto, 1997); e o esmero dos auditores em relação aos relatórios online (Sheehy e Trites, 1997). As companhias possuem diferentes políticas e práticas no uso da rede para divulgar relatórios financeiros. Em 1996, nos Estados Unidos, surgia o EDGAR (Electronic Data Gathering, Analysis, and Retrieval), uma tentativa de padronizar procedimentos, enquanto no Canadá era criado o SEDAR (System for Electronic Document Analysis and Retrieval).

Essa temática também tem despertado o interesse da academia. Assim, Gray e Debreceny (1997) examinaram empresas norte-americanas e concluíram que mais de $60 \%$ delas utilizam a Web para disseminação de informações financeiras. Na Finlândia, Lymer e Tallberg (1997) encontraram resultados similares. Porém, na Espanha, Gowthorpe e Amat (1999) identificaram somente 19\% das empresas adotantes dessa prática. Mas existem também investigações que vão além da simples disponibilização dos relatórios financeiros. O relacionamento com investidores pela Web foi objeto de pesquisa realizada por Heldin (1999), o qual concluiu que, na Suécia, apesar de $83 \%$ das empresas divulgarem informações em seu Website, somente uma minoria delas o fazia de forma estruturada, oferecendo informações julgadas importantes, em tempo real.

Outros temas, como conteúdo, tecnologia e suporte ao investidor, também foram alvo de pesquisa. Pirchegger e Wagenhofer (1999) compararam empresas austríacas e alemãs e concluíram que, ao menos por meio eletrônico, as primeiras prestam um melhor serviço que as últimas. Em se tratando dos determinantes da disseminação de informações financeiras na Web, Marston e Leow (1998), de acordo com um conjunto de empresas no Reino Unido, concluíram que o tamanho da empresa está positivamente correlacionado à disponibilização de informações financeiras no Website corporativo. Essa associação, porém, não foi uniforme entre os diversos setores industriais considerados nessa pesquisa, corroborada pelo trabalho de Craven e Marston (1999).

O poder público nesses países inclui a adoção de legislações, ou medidas de menor magnitude, para Websites corporativos. Embora menos freqüente, também é possível encontrar entidades locais que incluem em suas homepages relatórios financeiros, a versão integral ou abreviada do relatório anual e outros documentos. Ainda são divulgadas informações úteis para expressar as condições financeiras e o desempenho da empresa (estratégias de longo prazo, indicadores de desempenho, estatísticas da economia e evolução demográfica). Enquanto em alguns países existem determinações acerca da disponibilização de informações financeiras pela Internet, em outros a situação é completamente diferente (Hassan, Jaffar e Zain, 1999). No caso do Brasil, ainda não existem orientações ou regras para esse efeito.

Alguns estudos, como os de Isenmann e Lenz (2000), Kolk (1999) e Jones, Alabaster e Hetherington (1999), atestam que atualmente, na América Latina, os relatórios disponíveis ainda são dominados por publicações em mídia impressa, mas há uma tendência crescente pelo uso da Internet. Algumas experiências a esse respeito podem ser destacadas. Recentemente, Mendes-da-Silva e Alves (2004) encontraram uma correlação positiva e significativa entre a divulgação de informações e o Q de Tobin em empresas latino-americanas. Contudo, as principais características das experiências internacionais encontradas na literatura podem ser visualizadas resumidamente no Quadro 1 a seguir. 


\section{Quadro 1- Fatores re lacionados à disseminação de relatórios financeiros pela Internet identificados em estudos anteriores}

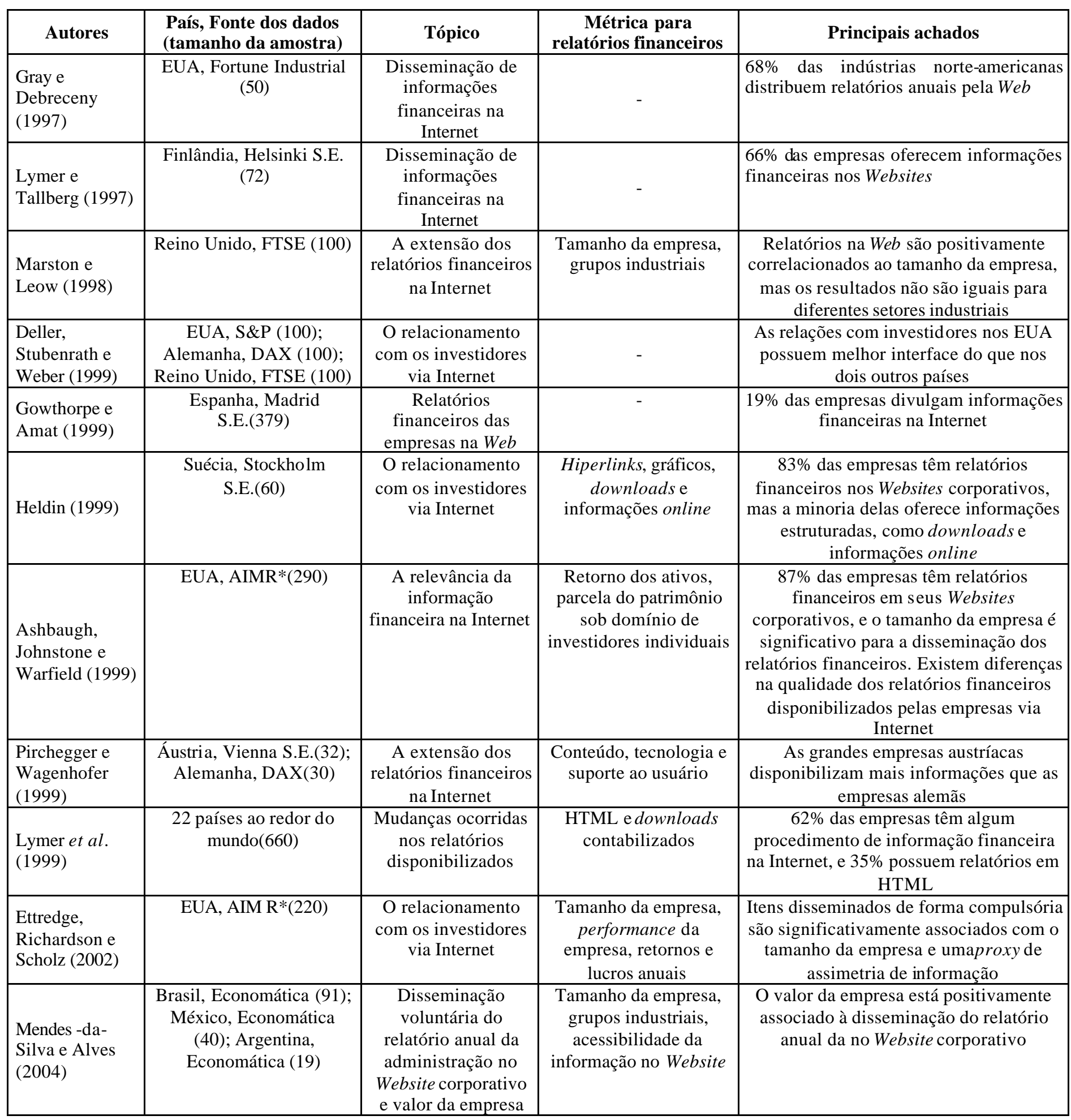

Nota: S.E. = Stock Exchange; * The Association for Investment and Research which provide analysis' ratings of overall disclosure quality. 


\section{DETERMINANTES DA DISSEMINAÇÃO VOLUNTÁRIA DE INFORMAÇÕES FINANCEIRAS NA INTERNET \\ Wesley Mendes-da-Silva - Paulo Azevedo de Oliveira Magalhães Filho}

Atualmente, nos países latino-americanos, especialmente em função da percepção de risco no mercado de ações, generalizada entre investidores, muitas empresas não conseguem obter uma valorização adequada. A principal razão para isso seria a falta de transparência e de garantias a investidores, assim como a própria ineficiência do mercado (incluindo-se a assimetria informacional). Evidências desse fenômeno foram encontradas em um estudo da McKinsey \& Company (2000), no qual se afirma que $83 \%$ dos investidores internacionais possuidores de recursos aplicados na América Latina estariam dispostos a pagar um prêmio por empresas com melhor governança corporativa.

\section{METODOLOGIA}

\section{Hipóteses}

A literatura acerca da disseminação voluntária de informações financeiras de forma geral, e particularmente na Internet, foi examinada para identificar quais características das empresas mais afetam a decisão de disseminar informações. Assim, as hipóteses desta pesquisa são formuladas com base em estudos anteriores, as quais são detalhadas a seguir.

Para Leftwich, Watts e Zimmerman (1981), a relação entre fornecedores externos de capital e os gerentes da empresa é um exemplo de relação de agência, na qual

os primeiros representam o principal e os últimos, os agentes. A teoria da agência, cuja origem remonta a discussão do conflito de interesses entre acionistas e gestores, discutido por Jensen e Meckling (1976), sugere que os custos de agência do capital emprestado dependem dos objetivos dos fornecedores externos de capital. Isso indica que os custos seriam mais altos para as empresas com maior alavancagem. Adicionalmente, Jensen e Meckling (1976) concluem que a disseminação voluntária pode reduzir os custos de agência por facilitar a avaliação da habilidade da empresa para contrair endividamento.

Enquanto alguns estudos revelaram relação positiva entre disseminação voluntária e a alavancagem financeira da empresa (Mitchell, Chia e Loh, 1995; Hossain, Berera e Rahman, 1995), outros não apóiam esses resultados. Assim, Mckinnon e Dalimunthe (1993), Aitken, Hooper e Pickering (1997) e Brennan e Hourigan (2000) não encontraram relação positiva significativa entre a alavancagem e o nível de disseminação de informações financeiras, até mesmo sugerindo que essa relação seja negativa e significativa (Meek, Roberts e Gray, 1995). Os resultados oferecidos por esses estudos não oferecem conclusões. Dessa forma, neste artigo testa-se a relação entre alavancagem e a disseminação voluntária de informações financeiras na Internet, e a primeira hipótese é a seguinte:

H1: Inexiste uma associação entre a alavancagem financeira da empresa e a disseminação de informações financeiras na Internet. 


\section{DETERMINANTES DA DISSEMINAÇÃO VOLUNTÁRIA DE INFORMAÇÕES FINANCEIRAS NA INTERNET \\ Wesley Mendes-da-Silva - Paulo Azevedo de Oliveira Magalhães Filho}

Todavia, investidores geralmente percebem a ausência de disseminação voluntária de informações como um indicador de "más notícias" sobre uma empresa. Assim, empresas possuidoras de desempenho superior teriam um incentivo adicional para disseminar informações voluntariamente (Lev e Penman, 1990; Lang e Lundholm, 1993; Clarkson, Kao e Richardson, 1994). Desse modo, a segunda hipótese deste estudo é:

H2: A quantidade de informação disseminada no Website da empresa é positivamente relacionada à sua performance.

Cox (1985), Waymire (1985), Lang e Lundholm (1993), Clarkson, Kao e Richardson (1994), entre outros, encontraram uma associação positiva entre o tamanho da empresa e a quantidade de disseminação voluntária. A hipótese então é:

H3: A quantidade de informações disseminadas no Website é positivamente relacionada ao tamanho da empresa.

Singhvi e Desai (1971) encontraram relacionamento positivo entre a rentabilidade e a qualidade da disseminação de informações. Assim, o gerente poderia ser encorajado a divulgar informações financeiras quando as taxas de rentabilidade fossem altas, expressando a boa qualidade da administração. Porém, segundo evidenciam os resultados obtidos por Ashbaugh, Johnstone e Warfield (1999), a relação entre a rentabilidade e a disseminação de informações financeiras seria insignificante. Como os resultados das pesquisas nesse campo são inconclusivos, isso motiva testar a seguinte hipótese:

H4: Inexiste uma associação entre a rentabilidade da empresa e a disseminação de informações financeiras na Internet.

Adiante serão expostos os procedimentos realizados para a constituição do conjunto de empresas estudadas, assim como a coleta de dados e as variáveis envolvidas.

\section{Amostra, dados e variáveis}

A composição da amostra foi determinada por alguns fatores. Primeiro, as observações para as variáveis dependentes, que proporcionaram a obtenção de um índice, uma proxy de disseminação, obtido pela visitação e exploração de cada Website corporativo. As variáveis dependentes são baseadas nas utilizadas por Ettredge, Richardson e Scholz (2002). Cada Website foi visitado duas vezes por 
pesquisadores diferentes, então as duas informações para cada empresa foram comparadas e as diferenças, reconciliadas.

Segundo, foram consideradas apenas as empresas não-financeiras (as 21 empresas financeiras listadas foram excluídas, por trabalharem essencialmente alavancadas) listadas na Bolsa de Valores de São Paulo (Bovespa) em junho de 2002. A amostra inicial compôs-se de 418 empresas, das quais 127 não mantinham Website, ou este não foi encontrado no banco de dados da Economática ${ }^{\circledR}$, nem tampouco no site de busca utilizado, o Google (www.google.com). Assim, o conjunto final de empresas estudadas foi composto de 291 empresas, pouco menos de $70 \%$ do conjunto inicial, distribuídas entre 18 setores de atividade, como ilustra a Tabela 1.

Tabela 1 -Conjunto de empresas estudadas por setores da economia

\begin{tabular}{lcc}
\hline \multicolumn{1}{c}{ Setor } & Freqüência & Percentual \\
\hline Telecomunicações & 35 & 12,0 \\
Outros & 33 & 11,3 \\
Siderurgia & 30 & 10,3 \\
Energia elétrica & 28 & 9,6 \\
Química & 24 & 8,2 \\
Têxtil & 20 & 6,9 \\
Alimentos & 18 & 6,2 \\
Veículos e peças & 18 & 6,2 \\
Eletroeletrônicos & 17 & 5,8 \\
Construção & 12 & 4,1 \\
Máquinas & 11 & 3,8 \\
Papel e celulose & 10 & 3,4 \\
Comércio & 9 & 3,1 \\
Petróleo & 9 & 3,1 \\
Minerais não-metálicos & 8 & 2,7 \\
Transporte & 5 & 1,7 \\
Mineração & 3 & 1,0 \\
Agropecuária & 1 & 0,3 \\
Total & 291 & 100,0 \\
\hline
\end{tabular}

As variáveis dependentes aqui utilizadas, $R E Q, V O L$ e INDICE, que estão definidas na Tabela 2, foram essencialmente constituídas segundo procedimento sugerido por Ettredge, Richardson e Scholz (2002), os quais, para tanto, realizaram uma série de entrevistas com diretores de relações com investidores nos Estados Unidos. 
Tabela 2 - Definição das variáveis dependentes

\begin{tabular}{|c|c|}
\hline Categoria & Descrição operacional \\
\hline \multicolumn{2}{|l|}{ arquivos requeridos } \\
\hline Relatório anual & $\begin{array}{l}=1 \text { se o site disponibilizar um relatório completo. Igual a } 0 \text { se a informação relativa } \\
\text { ao relatório anual não estiver disponível }\end{array}$ \\
\hline Relatório trimestral & $\begin{array}{l}=1 \text { se o site disponibilizar relatórios trimestrais incluindo relatórios financeiros. } \\
\text { Igual a } 0 \text { se não estiver disponível. }\end{array}$ \\
\hline Outros arquivos & $\begin{array}{l}\text { = } 1 \text { se outros documentos da CVM, além dos relatórios anuais ou trimestrais, } \\
\text { estiverem disponíveis no site (Relatório de Informações Anuais - IAN, por exemplo), } \\
\text { e } 0 \text { se não o disponibilizarem. }\end{array}$ \\
\hline Link para $C V M$ & $\begin{array}{l}=1 \text { se o site disponibilizar um link para o site da Comissão de Valores Mobiliários - } \\
\text { CVM, e } 0 \text { se não o disponibilizarem. }\end{array}$ \\
\hline$R E Q$ & = a soma dos escores das quatro características acima. \\
\hline \multicolumn{2}{|l|}{ Disseminação voluntária } \\
\hline Recente & $\begin{array}{l}=1 \text { se os dados financeiros mensais mais recentes estiverem no site, e } 0 \text { se não } \\
\text { estiverem. }\end{array}$ \\
\hline Visão geral & $\begin{array}{l}\text { = } 1 \text { se o site oferecer uma visão geral consistente do desempenho da empresa } \\
\text { (perguntas mais freqüentes, fatos recentes, notícias rápidas), e } 0 \text { se não a oferecer. }\end{array}$ \\
\hline Idioma & $\begin{array}{l}=1 \text { se a empresa disponibilizar as informações financeiras em outro idioma além do } \\
\text { português, e } 0 \text { se não as oferecer. }\end{array}$ \\
\hline Calendário & $\begin{array}{l}=1 \text { se o site proporcionar um calendário de eventos de interesse do investidor (por } \\
\text { exemplo, a data de distribuição dos dividendos), e } 0 \text { se não o proporcionar. }\end{array}$ \\
\hline Notícias & $\begin{array}{l}=1 \text { se o site oferecer o texto das notícias financeiras mais recentes, e } 0 \text { se não o } \\
\text { oferecer. }\end{array}$ \\
\hline Analistas & $=1$ se o site elencar analistas, e 0 se não o oferecer. \\
\hline Comentários & $\begin{array}{l}\text { = } 1 \text { se o site apresentar textos de comentários e apresentações (por exemplo, os road } \\
\text { shows do desempenho trimestral), e } 0 \text { se não os oferecer. }\end{array}$ \\
\hline Atual & $=1$ se o site fornecer os preços das ações no mesmo dia, e 0 se não os oferecer. \\
\hline Histórico & $=1$ se o site fornecer o histórico dos preços das ações, e 0 se não o oferecer. \\
\hline Link diferente & $\begin{array}{l}=1 \text { se o site oferecer um link para os dados das ações em um site diferente, e } 0 \text { se não } \\
\text { o oferecer. }\end{array}$ \\
\hline Corretora & $\begin{array}{l}=1 \text { se o site oferecer informação sobre a(s) sua(s) corretora(s) (endereço ou link } \\
\text { direto), e } 0 \text { se não a oferecer. }\end{array}$ \\
\hline Vantagem & $\begin{array}{l}=1 \text { se o site discutir as vantagens de um investidor em manter a posse das ações da } \\
\text { empresa. }\end{array}$ \\
\hline Reinvestimento & $\begin{array}{l}=1 \text { se o site fornecer informações detalhando um plano de reinvestimento de } \\
\text { dividendos e, } 0 \text { se não as oferecer. }\end{array}$ \\
\hline$V O L$ & = a soma dos escores das 13 características acima. \\
\hline INDICE & $=R E Q+V O L$ \\
\hline
\end{tabular}

Contudo, a variedade de características de Websites, juntamente com a literatura afeta ao tema, serviu de base para que esses autores estimassem o nível de disseminação de informações financeiras de interesse do investidor pela Internet. A opção por esse procedimento de avaliação do nível de 
disseminação de informações na Internet pode oferecer limitações a esta pesquisa, bem como aos seus resultados. Ademais, os aspectos verificados por Ettredge, Richardson e Scholz (2002) podem não refletir integralmente a demanda por informações corporativas pelos investidores brasileiros, ressaltando-se que ainda não existem pesquisas sobre essa temática.

Adicionalmente, para avaliar o direcionamento a mercados internacionais, neste artigo também foi verificada a disponibilização dos relatórios anuais em idiomas além do português. Assim, como sugerem Ettredge, Richardson e Scholz (2002), as características foram divididas em duas categorias: (a) arquivos requeridos $(R E Q)$, constituída pelos arquivos mais frequientemente solicitados pelos analistas ou investidores; e (b) disseminação voluntária $(V O L)$. Os dados necessários à obtenção de cada variável foram obtidos pela visitação dos Websites corporativos entre agosto e outubro de 2002.

O valor da variável $R E Q$ para uma empresa $i$ é igual à soma dos escores identificados para as características requeridas na categoria de relatórios verificada no site: Relatório anual, Relatório trimestral, Outros arquivos e Link para a CVM (foi verificada a existência de um link para o site da Comissão de Valores Mobiliários, CVM, porque este inclui muitas informações que podem não estar contempladas em relatórios anuais). Da mesma forma, a variável $V O L$ é igual à soma dos escores para as características consideradas nessas duas categorias. A variável INDICE para uma empresa $i$ consiste na soma dos valores obtidos para as variáveis $R E Q$ e $V O L$ nessa mesma empresa. A distribuição de freqüência das informações sobre os Websites corporativos encontra-se na Tabela 3.

Tabela 3 - Distribuição de freqüência da disseminação dos itens nos Websites corporativos

\begin{tabular}{rccc}
\hline Car acterística do site corporativo & Freqüência & $\begin{array}{c}\text { Percentual das empresas } \\
\text { que disseminaram }\end{array}$ & Categoria do item \\
\hline Relatório anual & 144 & 49,5 & REQ \\
Relatório trimestral & 115 & 39,5 & REQ \\
Outros arquivos & 108 & 37,1 & REQ \\
Visão geral & 106 & 36,4 & VOL \\
Notícias & 94 & 32,3 & VOL \\
Idioma & 86 & 29,6 & VOL \\
Comentários & 64 & 22,0 & VOL \\
Recente & 62 & 21,3 & VOL \\
Atual & 59 & 20,3 & VOL \\
Link diferente & 56 & 19,2 & VOL \\
Histórico & 50 & 17,2 & VOL \\
Calendário & 33 & 11,3 & VOL \\
Analistas & 33 & 11,3 & VOL \\
Corretora & 27 & 9,3 & VOL \\
Link para CVM & 24 & 8,2 & REQ \\
Vantagem & 16 & 5,5 & VOL \\
\hline Reinvestimento & 4 & 1,4 & VOL \\
\hline Ca1 & 53 & &
\end{tabular}

$\mathrm{N}=291$ 


\section{DETERMINANTES DA DISSEMINAÇÃO VOLUNTÁRIA DE INFORMAÇÕES FINANCEIRAS NA INTERNET \\ Wesley Mendes-da-Silva - Paulo Azevedo de Oliveira Magalhães Filho}

Após examinados os Websites, ficou evidenciada a pífia disseminação voluntária de informações financeiras por parte das empresas. A pontuação média (mediana) obtida foi de 3,71 $(2,00)$ dos 17 pontos disponíveis para a variável INDICE, como mostra a Tabela 4.

Tabela 4 - Estatísticas descritivas das variáveis dependentes

\begin{tabular}{|c|c|c|c|c|c|c|c|}
\hline \multirow{2}{*}{$\begin{array}{c}\text { Variável } \\
\text { dependente }\end{array}$} & \multirow{2}{*}{ Média } & \multirow{2}{*}{$\begin{array}{c}\text { Desvio } \\
\text { padrão }\end{array}$} & \multirow{2}{*}{ Mínimo } & \multirow{2}{*}{ Máximo } & \multicolumn{3}{|c|}{ Percentis } \\
\hline & & & & & $25 \%$ & Mediana & $75 \%$ \\
\hline$\overline{R E Q}$ & 1,340 & 1,457 & 0,000 & 4,000 & 0,000 & 1,000 & 3,000 \\
\hline$V O L$ & 2,370 & 3,248 & 0,000 & 12,000 & 0,000 & 1,000 & 4,000 \\
\hline INDICE & 3,710 & 4,487 & 0,000 & 16,000 & 0,000 & 2,000 & 7,000 \\
\hline
\end{tabular}

No conjunto das variáveis explanatórias, definidas na Tabela 5, o nível de participação de capitais de terceiros foi calculado a partir da alavancagem financeira da empresa, ALAV. A performance da empresa foi medida pelo retorno anual das ações mais líquidas da empresa, RETN, no ano anterior, 2001. O tamanho da empresa, $\ln T A M F$, foi mensurado pelo valor contábil do ativo total da empresa $i$, no final do ano anterior, 2001. A variável $R O A$ é indicada pela rentabilidade do ativo total de uma empresa $i$ no ano anterior.

As variáveis de controle $(V C)$ foram selecionadas com base na possível influência que exercem sobre as variáveis dependentes de divulgação voluntária de informações financeiras na Internet (REQ, VOL e INDICE) e explanatórias ( $A L A V, R E T N, \ln T A M F$ e ROA). Caso as variáveis de controle possuam forte correlação com algumas dessas variáveis e não sejam consideradas no modelo, a relação entre as variáveis de interesse pode não ser evidenciada de forma adequada. Assim, as seguintes variáveis de controle foram utilizadas: (a) liquidez em Bolsa de Valores (LIQB); (b) índice $Q$ de Tobin $(Q)$, proxy de valor da empresa; e (c) nível de governança corporativa na Bovespa (NGC). 
Tabela 5 - Definição de variáveis explanatórias

\begin{tabular}{|c|c|c|c|}
\hline Hipótese & Variável & Descrição & Fontes Conceituais* \\
\hline H1 & $A L A V$ & $\begin{array}{l}\text { Alavancagem financeira de uma empresa } i \text { no ano } t \text {-1 calculada } \\
\text { segundo a expressão: } \\
\qquad A L A V=\frac{L L}{P L} \\
\text { onde: } \\
\text { LL = Lucro líquido; PL = Patrimônio líquido; RF }=\text { Resultado } \\
\text { financeiro; AT = valor contábil do ativo total. }\end{array}$ & $\begin{array}{r}\text { Mckinnon e Dalimunthe } \\
\text { (1993); Michell, } \\
\text { Chia e Loh (1995); } \\
\text { Meek, Roberts e Gray } \\
\text { (1995); } \\
\text { Hossain, Berera e Rahman } \\
\text { (1995); Aitken, Hooper e } \\
\text { Pickering (1997); Brennan e } \\
\text { Hourigan (2000) }\end{array}$ \\
\hline $\mathrm{H} 2$ & RETN & $\begin{array}{l}\text { Retorno anual oferecido pelas ações mais líquidas da empresa } i \text { no } \\
\text { ano } t \text {-1, calculado pela expressão: } \\
\qquad R E T N=\left[\frac{P_{f}-P_{i}+\text { Dividendos }}{P_{i}}\right] \times 100 \\
\text { onde: } \\
P_{f}=\text { cotação da ação no último pregão do ano } t-1 ; \\
P_{i}=\text { cotação da ação no primeiro pregão do ano } t-1 .\end{array}$ & $\begin{array}{r}\text { Lev e Penman (1990); } \\
\text { Lang e Lundholm (1993); } \\
\text { Clarkson, Kao e Richardson } \\
\text { (1994); } \\
\text { Ettredge, Richardson e } \\
\text { Scholz (2002) }\end{array}$ \\
\hline $\mathrm{H} 3$ & $\ln T A M F$ & $\begin{array}{l}\text { Logaritmo natural do valor contábil do ativo total da empresa } i \text { no } \\
\text { ano } t \text {-1, expresso em milhares de reais. }\end{array}$ & $\begin{array}{r}\text { Cox (1985); Waymire } \\
\text { (1985); Lang e Lundholm } \\
\text { (1993); Ettredge, Richardson } \\
\text { e Scholz (2002). }\end{array}$ \\
\hline $\mathrm{H} 4$ & $R O A$ & $\begin{array}{l}\text { Retorno do ativo total da empresa } i \text { no ano } t-1 \text { calculado segundo a } \\
\text { expressão: } \\
\qquad R O A=\frac{L A J I R}{A T} \\
\text { onde: } L A J I R=\text { lucro antes dos juros e impostos; } \\
\quad A T=\text { valor contábil do ativo total. }\end{array}$ & $\begin{array}{r}\text { Singhvi e Desai (1971); } \\
\text { Ashbaugh, Johnstone e } \\
\text { Warfield (1999); Ismail } \\
\text { (2000) }\end{array}$ \\
\hline \multirow{3}{*}{ 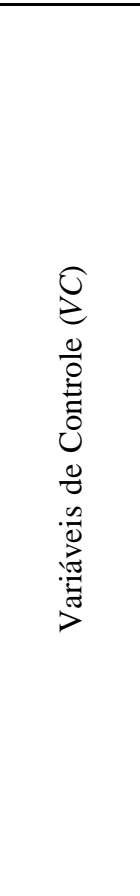 } & $L I Q B$ & $\begin{array}{l}\text { Liquidez em Bolsa calculada pela expressão: } \\
\qquad L I Q B=\frac{100 p \sqrt{n v / N V}}{P} \\
\text { onde: } p=\text { número de dias em que houve ao menos um negócio } \\
\text { com a ação de um período escolhido; } P=\text { número total de dias do } \\
\text { período escolhido; } n=\text { número de negócios com a ação dentro de } \\
\text { um período; } N=\text { número de negócios com todas as ações dentro de } \\
\text { um período; } v=\text { volume de dinheiro com a ação dentro do período } \\
\text { escolhido; } V=\text { volume de dinheiro com todas as ações dentro do } \\
\text { período escolhido. }\end{array}$ & \\
\hline & $Q$ & $\begin{array}{l}\text { Îndice } Q \text { de Tobin, medida de valor de mercado da companhia, que é } \\
\text { calculado pela expressão: } \\
\qquad Q=\left[\frac{V M O+V M A P+D I V T}{A T}\right] \\
\text { onde: } V M O=\text { valor de mercado das ações ordinárias; VMAP = } \\
\text { Valor de mercado das ações preferenciais; } D I V T=\text { valor contábil } \\
\text { das dívidas de curto e longo prazo menos o ativo circulante após a } \\
\text { exclusão do valor dos estoques; } A T=\text { valor contábil do ativo total }\end{array}$ & Chung e Pruitt (1994) \\
\hline & $N G C$ & $\begin{array}{l}\text { Variável dicotômica que expressa se a empresa encontra-se listada } \\
\text { nos Níveis de Governança Corporativa da Bovespa. Recebeu valor } \\
=1 \text { se pertence ao Novo Mercado, Nível } 1 \text { ou Nível } 2 \text {; e valor }=0 \\
\text { nos casos alternativos. }\end{array}$ & \\
\hline
\end{tabular}

*Fonte operacional: banco de dados da Economática®. 


\section{Modelos empíricos}

$\mathrm{Na}$ análise multivariada, dois modelos foram testados. Primeiro, tendo as variáveis dependentes $R E Q$, $V O L$ e INDICE, utilizourse o modelo ilustrado na equação (1). Assim, quatro variáveis explanatórias contínuas foram adotadas para testar as hipóteses propostas, H1, H2, H3 e H4. Então, assumiu-se que a soma das informações financeiras disponibilizadas por uma empresa $i$ em seu Website corporativo como função de $A L A V, R E T N, \ln T A M F$ e de ROA.

$$
\operatorname{INDICE}_{i}=\delta_{0}+\delta_{1} A L A V_{i}+\delta_{2} \operatorname{RETN}_{i}+\delta_{3} \ln T A M F_{i}+\delta_{4} R O A_{i}+\sum_{j=1}^{k} \psi_{j} V C_{j i}+\xi_{i}
$$

A partir da proposição de Botosan (1997, p. 329) de que, entre as características relacionadas na Tabela 2, os relatórios anuais seriam as informações mais relevantes para o mercado, constituindo-se em uma boa proxy de disseminação voluntária, adotou-se o procedimento de verificar as associações entre as variáveis explanatórias utilizadas no estudo e a probabilidade, $\Pi_{i}$, de disseminação dos relatórios anuais no Website. Para tanto, empregourse um modelo de regressão logística, o qual é ilustrado pela equação (2).

$$
\ln \left(\frac{\Pi_{i}}{1-\Pi_{i}}\right)=\eta_{0}+\eta_{1} A L A V_{i}+\eta_{2} \text { RETN }_{i}+\eta_{3} \ln T A M F_{i}+\eta_{4} R O A_{i}+\sum_{j=1}^{k} \lambda_{j} V C_{j i}+v_{i}
$$

Onde, $\Pi=\left(\frac{1}{1+e^{-\left(\eta_{0}+\eta_{1} A L A V_{i}+\eta_{2} R E T N_{i}+\eta_{3} \ln T A M F_{i}+\eta_{4} R O A_{i}+\sum_{j=1}^{k} \lambda V C_{j i}\right)}}\right)$

\section{RESULTADOS}

Os principais resultados obtidos na regressão múltipla entre as características da empresa e as variáveis dependentes de disseminação de informações financeiras na Internet estão resumidos na Tabela 6. A estatística Durbin-Watson $(d)$ revelou não existir autocorrelação de primeira ordem entre os resíduos das regressões para as variáveis $R E Q$ e $V O L$. Porém, mostrourse inconclusiva a esse respeito para a variável INDICE, já que, para esta última variável, assumiu um valor que está incluído no intervalo que assume a possibilidade da existência de correlação serial de primeira ordem, como ensinam Savin e White (1977).

Entretanto, Granger e Newbold (1974) defendem que uma boa regra prática para suspeitar de que a regressão estimada sofre de regressão espúria seria $u m \mathrm{R}^{2}>d$. Os coeficientes de determinação, $\mathrm{R}^{2}$ ajustado, ficaram acima de 0,33, enquanto Ettredge, Richardson e Scholz (2002) obtiveram um máximo de apenas 0,175 .

Os resultados revelaram uma associação negativa entre o retorno das ações da empresa e a quantidade de informações disseminadas na $W e b$, o que oferece motivo para a rejeição de $\mathrm{H} 2$. Isso pode sugerir uma busca das empresas pela redução da assimetria informacional com relação à 


\section{DETERMINANTES DA DISSEMINAÇÃO VOLUNTÁRIA DE INFORMAÇÕES FINANCEIRAS NA INTERNET \\ Wesley Mendes-da-Silva - Paulo Azevedo de Oliveira Magalhães Filho}

precificação de seus títulos ou mesmo o oferecimento de dados para o mercado, os quais poderiam reduzir seus custos de capital. No entanto, a proxy de valor da empresa, o $Q$ de Tobin, não se mostrou significativamente associado às variáveis independentes. As empresas de maior porte $(\ln T A M F)$ revelaram uma maior quantidade de informações disponibilizadas no Website corporativo, o que não possibilitou a rejeição de H3.

Tabela 6 - Parâmetros estimados para o modelo de regressão múltipla

\begin{tabular}{|c|c|c|c|c|c|c|}
\hline $\begin{array}{c}\text { Variáveis } \\
\text { Dependentes }\end{array}$ & Hipóteses & $\begin{array}{c}\text { Variáveis } \\
\text { Independentes }\end{array}$ & $\begin{array}{c}\text { Sinal } \\
\text { Esperado }\end{array}$ & $\begin{array}{c}\text { Coeficientes } \\
\text { Padronizados (Beta) }\end{array}$ & $\begin{array}{c}\text { Estatística t } \\
\left(\mathrm{R}^{2} \text { ajustado }\right) \\
{[d]}\end{array}$ & Sig. \\
\hline \multirow{10}{*}{$R E Q$} & \multirow{10}{*}{$\begin{array}{l}\mathrm{H} 1 \sqrt{ } \\
\mathrm{H} 2 \times \\
\mathrm{H} 3 \sqrt{ } \\
\mathrm{H} 4 \sqrt{ }\end{array}$} & (Constant) & & & $-3,429$ & 0,001 \\
\hline & & $A L A V$ & $?$ & $-0,057$ & $-0,856$ & 0,393 \\
\hline & & $R E T N$ & positivo & $-0,242$ & $-3,526$ & 0,001 \\
\hline & & $\ln T A M F$ & positivo & 0,350 & 4,561 & 0,000 \\
\hline & & $R O A$ & $?$ & 0,063 & 0,931 & 0,353 \\
\hline & & $L I Q B$ & & 0,197 & 2,457 & 0,015 \\
\hline & & & & 0,076 & 1,037 & 0,301 \\
\hline & & $N G C$ & & 0,175 & 2,678 & 0,008 \\
\hline & & & & & $(0,342)$ & \\
\hline & & & & & {$[1,902]$} & \\
\hline \multirow{10}{*}{$V O L$} & \multirow{10}{*}{$\begin{array}{l}\mathrm{H} 1 \sqrt{ } \\
\mathrm{H} 2 \boldsymbol{x} \\
\mathrm{H} 3 \sqrt{ } \\
\mathrm{H} 4 \sqrt{ }\end{array}$} & (Constant) & & & $-3,595$ & 0,000 \\
\hline & & ALAV & $?$ & $-0,007$ & $-0,098$ & 0,922 \\
\hline & & RETN & positivo & $-0,143$ & $-2,073$ & 0,040 \\
\hline & & $\operatorname{lnTAMF}$ & positivo & 0,330 & 4,288 & 0,000 \\
\hline & & ROA & $?$ & 0,021 & 0,318 & 0,751 \\
\hline & & LIQB & & 0,196 & 2,427 & 0,016 \\
\hline & & $\mathrm{Q}$ & & 0,081 & 1,093 & 0,276 \\
\hline & & NGC & & 0,256 & 3,894 & 0,000 \\
\hline & & & & & $(0,336)$ & \\
\hline & & & & & {$[1,860]$} & \\
\hline \multirow{9}{*}{ INDICE } & \multirow{9}{*}{$\begin{array}{l}\text { H1 } \\
\text { H2 } x \\
\text { H3 } \sqrt{ } \\
\text { H4 }\end{array}$} & (Constant) & & & $-3,821$ & 0,000 \\
\hline & & $A L A V$ & $?$ & $-0,025$ & $-0,378$ & 0,706 \\
\hline & & RETN & positivo & $-0,185$ & $-2,761$ & 0,006 \\
\hline & & $\ln T A M F$ & positivo & 0,354 & 4,727 & 0,000 \\
\hline & & $R O A$ & $?$ & 0,037 & 0,564 & 0,574 \\
\hline & & $L I Q B$ & & 0,206 & 2,631 & 0,009 \\
\hline & & $Q$ & & 0,083 & 1,160 & 0,248 \\
\hline & & $N G C$ & & 0,240 & $\begin{array}{c}3,767 \\
(0,373)\end{array}$ & 0,000 \\
\hline & & & & & {$[1,780]$} & \\
\hline
\end{tabular}

Fonte: Dados da pesquisa; $\mathrm{N}=164$. Nota: Software estatístico SPSS ${ }^{\circ}$ versão 11.0; Método Enter. DurbinWatson $[d]$ entre colchetes; $\mathrm{R}^{2}$ ajustado entre parênteses. $\mathbf{x}$ hipótese rejeitada; $\sqrt{ }$ hipótese não rejeitada.

A interação entre essa variável dependente e RETN, e também com lnTAMF, pode ser mais bem observada nos Gráficos 1 e 2, respectivamente. Essa associação pode ser conseqüência de um efeito de escala, à medida que a empresa cresce e torna-se mais exigida do ponto de vista da divulgação de informações para o mercado, e daí a verificação desse resultado. Tanto a liquidez em Bolsa de Valores $(L I Q B)$ como o nível de governança corporativa das empresas $(N G C)$ mostraram-se positivamente associados às variáveis dependentes. 


\section{Gráfico 1 - Relacionamento entre o retorno anual das ações e índice de disseminação de informações financeiras no Website}

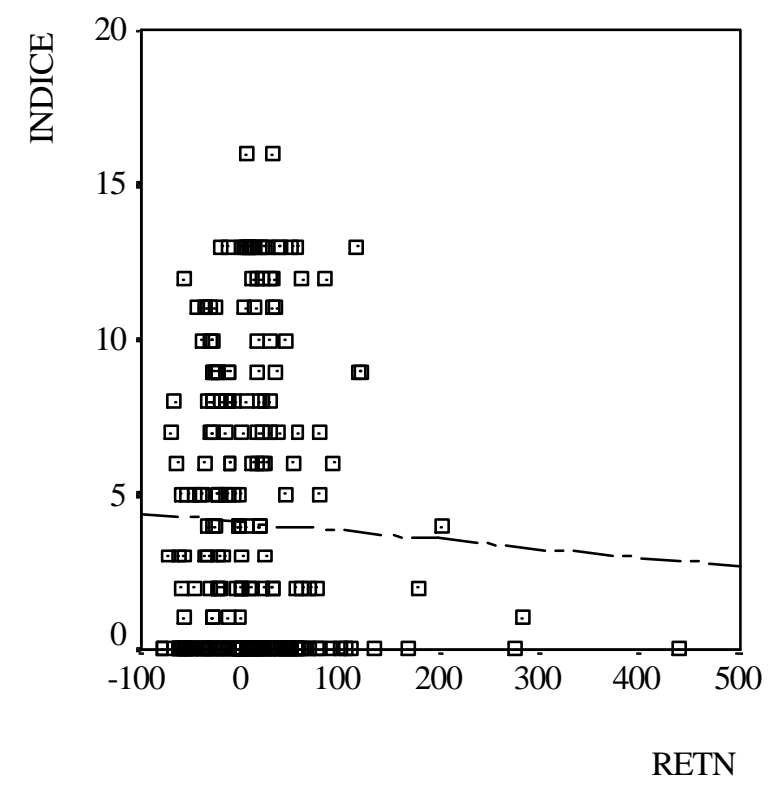

\section{Gráfico 2 - Relacionamento entre o tamanho da empresa e o índice de disseminação de informações financeiras no Website}

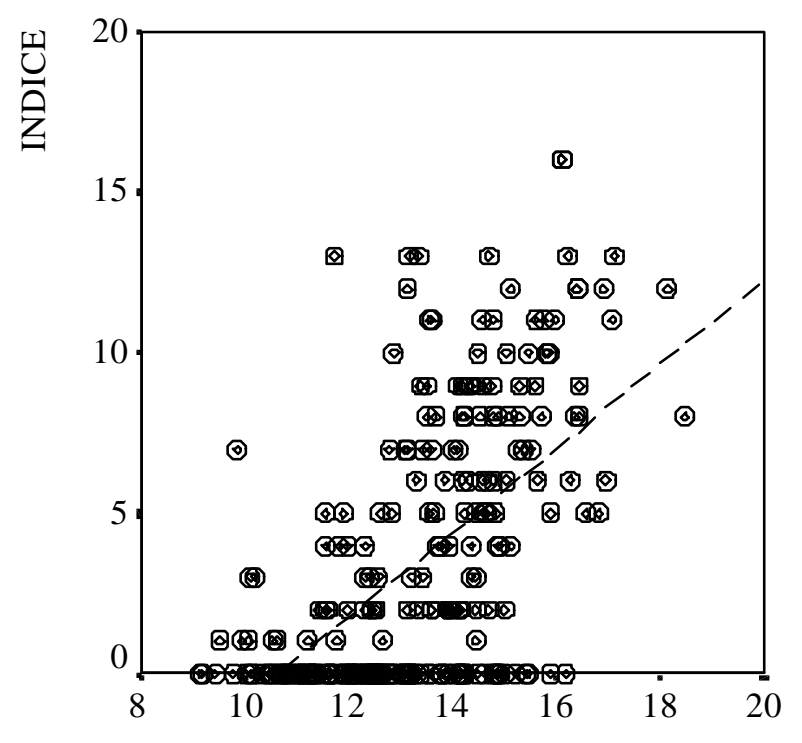

$\operatorname{lnTAMF}$

Fonte: Dados da pesquisa.

Esse resultado não é surpreendente, já que, como ilustra a Tabela 7, existe uma correlação significativa a $1 \%$ entre o tamanho da empresa, a liquidez em Bolsa e o nível de governança corporativa da empresa na Bovespa. É conveniente ressaltar a correlação relativamente alta entre $L I Q B$ e $\ln T A M F$, podendo sugerir a ocorrência de multicolinearidade, que pode ter como principais efeitos a rejeição da hipótese nula equivocadamente; sinais errados para os coeficientes estimados; ou mesmo a flutuação demasiada dos coeficientes da regressão entre amostras diferentes. Em síntese, se as variáveis explanatórias são fortemente correlacionadas, o modelo pode não estar adequado, incluindo tais variáveis. Um procedimento de mensuração da multicolinearidade utiliza o Fator Inflacionário da Variância (FIV) para cada variável explanatória; o critério mais conservador utilizado é o sugerido por Snee (1973). 


\section{DETERMINANTES DA DISSEMINAÇÃO VOLUNTÁRIA DE INFORMAÇÕES FINANCEIRAS NA INTERNET \\ Wesley Mendes-da-Silva - Paulo Azevedo de Oliveira Magalhães Filho}

Tabela 7 - Matriz de correlação entre as variáveis explanatórias

\begin{tabular}{|c|c|c|c|c|c|c|c|}
\hline & $A L A V$ & RETN & $\ln T A M F$ & $R O A$ & $L I Q B$ & $Q$ & $N G C$ \\
\hline$A L A V$ & 1 & & & & & & \\
\hline RETN & 0,052 & 1 & & & & & \\
\hline $\ln T A M F$ & $-0,087$ & 0,094 & 1 & & & & \\
\hline$R O A$ & $-0,011$ & $0,151 *$ & $0,246 * *$ & 1 & & & \\
\hline$L I Q B$ & 0,002 & 0,062 & $0,474 * *$ & $0,135^{*}$ & 1 & & \\
\hline$Q$ & $-0,145^{*}$ & $-0,007$ & $0,269 * *$ & $-0,028$ & $0,381 * *$ & 1 & \\
\hline$N G C$ & 0,002 & 0,004 & $0,245 * *$ & 0,042 & 0,033 & $-0,002$ & 1 \\
\hline
\end{tabular}

N mínimo = 164; N Máximo = 291; * Sig. < 0, 05; ** Sig. < 0,01.

$\mathrm{Na}$ óptica desse autor, se as variáveis explanatórias forem altamente correlacionadas, o FIV é superior a 5. Mas, nesse caso, os valores encontrados para o FIV encontram-se entre 1,004 e 1,351. Assim como era esperado para o caso brasileiro, a alavancagem financeira ( $A L A V)$ da empresa não se mostrou associada à quantidade de informações financeiras disponibilizadas na Internet, não possibilitando a rejeição de H1. A rentabilidade do ativo também não se mostrou associada às variáveis dependentes, o que não rejeita a hipótese H4. Esse resultado sugere a independência da divulgação de informações financeiras em relação à lucratividade da empresa. Ademais, de acordo com Ashbaugh, Johnstone e Warfield (1999), essa relação poderia ser positiva e não-significativa, o que é consistente com os resultados da regressão múltipla resumidos na Tabela 6.

Para estimar a probabilidade da divulgação do relatório anual, realizou-se uma regressão logística com 144 casos de divulgação e 147 observações para a situação alternativa. Dois testes estatísticos são apontados por Hair et al. (1998, p. 314-25) para avaliar a significância do modelo final, sendo que o primeiro refere-se ao Chi-quadrado para a mudança do valor do log da verossimilhança do modelo básico, o qual é comparável ao teste-F utilizado na análise de regressão múltipla. Além dessa medida, o indicador de Hosmer e Lemeshow $\left(\chi^{2}=5,178\right.$; $\mathrm{gl}=8$; Sig $\left.>0,05\right)$ indica que não foi estatisticamente significativa a diferença entre as classificações observadas e as preditas. Essas duas medidas combinadas fornecem subsídios para a avaliação do modelo gerado pela regressão logística ilustrado na Tabela 8.

Uma das vantagens da regressão logística é que se necessita apenas saber se um evento ocorreu para usar um valor dicotômico como variável dependente. A partir disso, a equação prediz informando a probabilidade de o evento ocorrer ou não. Então, se o valor da probabilidade for superior a 0,50, o caso será classificado no grupo dos relatórios anuais publicados no Website; no caso alternativo, é considerado como um dos não-publicados. 
Tabela 8 - Parâmetros estimados para o modelo de regressão logística

\begin{tabular}{ccccccc}
\hline $\begin{array}{c}\text { Variáveis } \\
\text { explanatórias }\end{array}$ & B & Erro padrão & Wald & gl & Sig. & Exp(B) \\
\hline RETN & $-0,013$ & 0,005 & 6,645 & 1 & 0,010 & 0,987 \\
$\ln T A M F$ & 0,544 & 0,142 & 14,688 & 1 & 0,000 & 1,723 \\
$L I Q B$ & 2,654 & 1,474 & 3,239 & 1 & 0,072 & 14,208 \\
Constante & $-7,179$ & 1,873 & 14,693 & 1 & 0,000 & 0,001 \\
\hline
\end{tabular}

Nota: Software estatístico utilizado: SPSS® versão 11.0.

Variável dependente: disponibilização dos relatórios anuais; $\chi^{2}=48,019 ; \mathrm{Sig}<0,01, \mathrm{gl}=7, \mathrm{~N}=164$; $\mathrm{R}^{2}$ de Cox Snell = 0,239; $\mathrm{R}^{2}$ de Nagelkerke = 0,341; Hosmer e Lemershow $\left(\chi^{2}=5,178 ; \mathrm{gl}=8\right.$; Sig. > 0,05); Método Foward Stepwise.

O teste Chi-Quadrado do modelo atesta se os coeficientes para todas as variáveis explanatórias, exceto o termo constante, é zero. O resultado obtido $\left(\chi^{2}=48,019\right.$; Sig $\left.<0,01\right)$ indica que os estimadores adotados podem distinguir adequadamente as companhias que divulgam relatórios anuais no Website, ao nível de significância de $1 \%$.

A Tabela 8 ilustra os coeficientes Betas estimados para as variáveis explanatórias significativas. A estatística Wald foi usada para testar se cada coeficiente é zero. Verificando o nível de significância para a estatística Wald, constata-se que o retorno das ações (RETN) contribuiu negativamente para a probabilidade de uma empresa publicar seu relatório anual no site. De forma inversa, o tamanho da empresa $(\ln T A M F)$ contribuiu positivamente para a divulgação desses relatórios, destacando-se o valor da estatística Exp (B), a qual revela quanto cada variável explanatória influencia na ocorrência da disseminação do relatório anual. Adicionalmente, a liquidez das ações da empresa mostrou-se positivamente correlacionada à divulgação dos relatórios anuais, sugerindo o efeito da demanda adicional por informações acerca das empresas possuidoras das ações mais freqüentemente negociadas, o que é corroborado pela significância da liquidez das ações da empresa (LIQB) no modelo.

Em contrapartida, os testes realizados para a regressão logística revelaram log da verossimilhança final de 175,194 (o inicial foi de 223,213). A par de que, quanto maior for o decaimento do $\log$ da verossimilhança melhor, o modelo não parece ter atingido resultados de ajuste suficientemente expressivos para referendá-lo, ao menos à luz das recomendações de Hair et al. (1998) a esse respeito. Destaque-se o valor do $\mathrm{R}^{2}$ de Nagelkerke 0,384 , uma estatística que varia de 0 a 1 , cujo papel desempenhado na regressão logística, é semelhante ao $\mathrm{R}^{2}$ da regressão múltipla, o que reforça a validade do modelo. Em síntese, com exceção do $\log$ da verossimilhança, o modelo proposto parece ter um ajuste razoável para a predição da disseminação do relatório anual no Website corporativo. O resultado de todas essas estatísticas reflete-se no Quadro 1, um percentual global de acertos de mais de $73 \%$ dos casos avaliados. 


\section{Quadro 1 - Resultados da classificação da disseminação do relatório anual}

\begin{tabular}{|c|c|c|c|c|}
\hline \multicolumn{2}{|c|}{ Observado } & \multicolumn{3}{c|}{ Predito } \\
\cline { 2 - 5 } & \multicolumn{2}{|c|}{$\begin{array}{c}\text { se existe relatório anual completo no site } \\
\text { não disponibiliza } \\
\text { relatório anual no site }\end{array}$} & $\begin{array}{c}\text { disponibiliza relatório } \\
\text { anual no site }\end{array}$ & $\begin{array}{c}\text { Percentagem de } \\
\text { acertos do modelo }\end{array}$ \\
\hline $\begin{array}{c}\text { Se disponibiliza } \\
\text { relatório anual no site } \\
\text { (contagem) }\end{array}$ & $\begin{array}{c}\text { não disponibiliza } \\
\text { relatório anual no site }\end{array}$ & 44 & 25 & $63,8 \%$ \\
\cline { 2 - 5 } & $\begin{array}{c}\text { disponibiliza relatório } \\
\text { anual no site }\end{array}$ & 19 & 76 & $80,0 \%$ \\
\hline Percentagem global & & & $\mathbf{7 3 , 2 \%}$ \\
\hline
\end{tabular}

Nota: O valor de corte para a classificação é 0,50 .

\section{CONCLUSÕES}

Em primeiro lugar, é importante destacar a falta de regras ou orientações acerca da utilização da Internet como meio de disseminação de informações financeiras por parte das empresas brasileiras. Observou-se isso no estudo e, mesmo sendo unânime tratar-se de um meio de comunicação com o mercado menos dispendioso que os métodos tradicionais, poucas empresas utilizam adequadamente a Web para tal. Isto é, quiçá a metade delas a utilizam. Ora, se uma empresa possui capital aberto ao mercado, quais seriam as razões para não disponibilizar informações acerca de sua realidade para o público de interessados usando a rede?

Entre as variáveis consideradas no modelo empírico proposto, o tamanho da empresa parece ser a que mais contribui para a disseminação voluntária de informações financeiras na Internet, o que pode ser uma indicação de um efeito de escala. Ou melhor, à medida que a empresa cresce, assume uma organização mais complexa, já que parece necessitar atender à demanda por informações por parte de investidores e credores. Aliado a essa evidência, observoutse que a liquidez em Bolsa e a inclusão em níveis diferenciados de governança corporativa também estão positivamente associadas à quantidade de informações financeiras disponibilizadas. No entanto, o retorno anual das ações das empresas, ao contrário das expectativas, revelou-se negativamente associado à quantidade de informações disponíveis no Website corporativo. Esse resultado pode sugerir uma busca pela redução da assimetria informacional acerca dos títulos das empresas. Isso provavelmente aumentaria a procura pelos papéis negociados pelas empresas, em decorrência da percepção de risco minimizado por parte do investidor, ou mesmo contribuiria para a redução do custo de capital, via credores e acionistas.

Indubitavelmente, a Internet constitui um meio veloz e pouco dispendioso para que as empresas desenvolvam relacionamento com o mercado aberto. Porém, diante das mudanças ocorridas nos meios de comunicação, não parece ser atraente aos olhos de alguns dos administradores das grandes empresas brasileiras, pelo menos à luz do que revelam os resultados. Assim, à vista das vantagens da ferramenta, 
torna-se imperativo desenvolver processos de utilização da rede mundial de computadores para relacionamento corporativo com o mercado.

As questões anteriormente colocadas juntam-se a outras e sugerem um campo promissor para estudos futuros, destacadamente aqueles que viabilizem a criação de novos modelos teóricos ou empŕicos, considerando um número cada vez maior de variáveis, já que as organizações estão tornando-se mais complexas, tendo em vista a sua inserção em mercados gradativamente mais competitivos e exigentes, ávidos por informação.

Embora este estudo tenha objetivado verificar a existência de associações entre características corporativas e a disseminação voluntária de informações financeiras usando a Internet, considerando apenas as companhias não financeiras listadas na Bovespa, em um dado período, o que pode limitar a generalização dos resultados obtidos, alguns aspectos podem ser citados como motivo de pesquisas futuras: identificar as razões pelas quais algumas empresas ainda não utilizam a Internet como meio de divulgação de informações financeiras; verificar, no âmbito nacional, segundo a opinião dos investidores e demais stakeholders, o perfil da demanda por informações financeiras das empresas pela Web; verificar a validade das informações financeiras divulgadas na Internet; discutir o aumento do uso da Internet para o relacionamento com investidores no âmbito das empresas brasileiras e suas implicações nos padrões de relatórios contábeis; investigar as associações entre investimento em Tecnologia de Informação e disseminação de informações financeiras na Internet; investigar as associações entre disseminação de informações financeiras na Internet e as estruturas de governança corporativa adotadas pelas empresas; analisar os impactos da disseminação voluntária de informações no custo dos capitais próprio e de terceiros utilizados pelas empresas.

\section{REFERÊNCIAS}

AITKEN, M.; HOOPER, C.; PICKERING, J. Determinants of Voluntary Disclosure of Segment Information: A Re-examination of the Role of Diversification Strategy, Accounting and Finance, v. 37, n. 1, p. 89-109, 1997.

ASHBAUGH, H.; JOHNSTONE, K. M.; WARFIELD, T. D. Corporate Reporting on the Internet. Accounting Horizons, Sept., v. 13, n. 3, p. 241-57, 1999.

BOTOSAN, C. A. Disclosure Level and the Cost of Equity Capital. The Accounting Review, v. 71, n. 3, July, p. 323-49, 1997.

BRENNAN, N.; HOURIGAN, D. Corporate Reporting on the Internet by Irish Companies. The Irish Accounting Review, v. 7, n. 1, p. 37-68, 2000.

CHUNG, K. H.; PRUITT, S. W. A Simple Approximation of Tobin's q. Financial Management, v. 23, n. 3, p. 70-4, Aut. 1994.

CLARKSON, P. M.; KAO, J. L.; RICHARDSON, G. D. The Voluntary Inclusion of Forecasts in the MD\&A Section of Annual Reports. Contemporary Accounting Research, v. 11, n. 1, p. 423-50, 1994. 
COX, C. T. Further Evidence on the Representativeness of Management Earning Forecasts. The Accounting Review, Oct., p. 692-701, 1985.

CRAVEN, B. N.; MARSTON, C. L. Financial reporting on the internet by leading UK companies. European Accounting Review, v. 8. n. 2, p. 321-33, 1999.

DEBRECENY, R.; GRAY, G. Financial reporting on the Internet and the external audit. The European Accounting Review, v. 8, n. 2, p. 335-50, 1999.

DELLER, D.; STUBENRATH, M.; WEBER, C. A Survey on the Use of the Internet for Investor Relations in the USA, the UK and Germany. European Accounting Review, v. 8, n. 2, p. 351-64, 1999.

DYCK, A.; ZINGALES, L. The Corporate Governance Role of the Media. Working Paper 9309. National Bureau of Economic Research, Cambridge, Massachussetts, 2002.

ETTREDGE, M.; RICHARDSON, V.; SCHOLZ, S. Dissemination of Information for Investors at Corporate Web Sites. Journal of Accounting and Public Policy, v. 21, p. 357-69, 2002.

GRAY, G. L.; DEBRECENY, R. S. Corporate Reporting on the Internet: Opportunities and Challenges. In ASIAN-PACIFIC CONFERENCE ON INTERNATIONAL ACCOUNTING ISSUES, $7^{\text {th }}, 1997$, Bangkok, Anais. Bangkok : Iai, 1997.

GRANGER, C. W. J.; NEWBOLD, P. Spurious Regressions in Econometrics. Journal of Econometrics, v. 2, p. 111-20, 1974.

GOWTHORPE, C.; AMAT, O. External reporting of accounting and financial internation via the Internet in Spain. The European Accounting Review. v. 8, n. 2, p. 365-71,1999.

HAIR, J. F. et al. Multivariate Data Analysis. New Jersey: Prentice Hall, 1998.

HASSAN, S.; JAFFAR, N.; ZAIN, M. M. Financial Reporting on the Internet by Malaysian Companies: Perceptions and Practices, Asia Pacific Journal of Accounting, v. 6, n. 2, 1999.

HELDIN, P. The Internet as a Vehicle for Investor Relations: The Swedish Case. European Accounting Review, v. 8, n. 2, p. 373-9, 1999.

HOSSAIN, M.; BERERA, M.; RAHMAN, A. Voluntary Disclosure in the Annual Reports of New Zealand Companies, Journal of International Financial Management and Accounting, v. 6, n. 1, p. 6987, 1995.

ISENMANN, R.; LENZ, C. System of Technical Benefits Using the Internet for Corporate Environmental Reporting. The 2000 Business Strategy and the Environment Conference. ERP Environment (Ed.). ERP: Shipley (UK), p. 203-10, 2000.

ISENMANN, R.; LENZ, C. Internet-Based Integration of Financial and Environmental reporting: Step Towards Sustentainability Reporting. The 2001 Business Strategy and Enviroment Conference. September $10^{\text {th }}$ and $11^{\text {th }}$, University of Leeds, UK, eds. By ERP Environment, Shipley (UK) 2001, p. 169-78, 2001. 


\section{DETERMINANTES DA DISSEMINAÇÃO VOLUNTÁRIA DE INFORMAÇÕES FINANCEIRAS NA INTERNET \\ Wesley Mendes-da-Silva - Paulo Azevedo de Oliveira Magalhães Filho}

ISMAIL, T. H. An Empirical Investigation of Factors Influencing Voluntary Disclosure of Financial Information on the Internet in the GCC Countries: artigo científico [2000]. Disponível em: <http://www.ssrn.com>. Acesso em: 23 set. 2003.

JENSEN, M. C.; MECKLING, W. Theory of firm: managerial behavior, agenc y costs and ownership structure. Journal of Financial Economics, v. 3, p. 305-60, Oct. 1976.

JONES, K.; ALABASTER, T.; HETHERINGTON, K. Internet-Based Environmental Reporting: Current Trend, 1999.

KOLK, A. The Internet as a Green Management Tool. Harvard Business Review, v. 6, n. 3, p. 307-16, 1999.

KORETO, R. J. If You Build It, Will They Come? Journal of Accountancy, v. 184, n. 1, p. 84-90, 1997.

LA PORTA, R.; LOPEZ-DE-SILANES, F.; SHLEIFER, A.; VISHNY, R. Law and Finance. Journal of Political Economy, v. 106, n. 6, p. 1113-1155, 1998.

LANG, M.; LUNDHOLM, R. Cross-Sectional Determinants of Analyst Ratings of Corporate Disclosures. Journal of Accounting Research, v. 31, n. 2, p. 246-71, 1993.

LEFTWICH, R.; WATTS, R.; ZIMMERMAN, J. Voluntary Corporate Disclo sure: The Case of Interim Reporting, Journal of Accounting Research, v. 19, p. 50-77, 1981.

LEV, B.; PENMAN, S. Voluntary Forecast Disclosure, Nondisclosure and Stock Prices. Journal of Accounting Research, v. 28, n. 1, p. 49-76, 1990.

LITAN, R. E.; WALLISON, P. J. The GAAP Gap: Corporate Disclosure in the Internet Age. Washington, DC: AEI-Brookings Joint Center for Regulatory Studies; La Vergne, Tenn, 2000.

LYMER, A.; TALLBERG, A. Corporate Reporting and the Internet - A Survey and Commentary on the Use of the WWW in Corporate Reporting in the UK and Finland. In ANNUAL CONGRESS OF THE EUROPEAN ACCOUNTING ASSOCIATION, 20², 1997, Graz, Anais. Graz : Eaa, 1997.

LYMER, A. The Internet and the Future of Corporate Reporting in Europe. European Accounting Review, v. 8, n. 2, p. 289-301, 1999.

MARSTON, C.; LEOW, C. Y. Financial Reporting on the Internet by Leading UK Companies. In EUROPEAN ACCOUNTING ASSOCIATION CONFERENCE, $21^{\text {st }}, 1998$, Belgium, Anais. Belgium: Eaa, 1998.

McKINSEY \& COMPANY. Investor Opinion Survey on Corporate Governance. June, 2000.

McKINNON, J.; DALIMUNTHE, L. Voluntary Disclosure of Segment Information by Australian Diversified Companies. Accounting and Finance, v. 33, n. 1, p. 33-50, 1993.

MEEK, G.; ROBERTS, C.; GRAY, S. Factors influencing Voluntary annual Report Disclosures by U.S., U.K. and Continental European Multinational Corporations, Journal of International Business Studies, v. 26, n. 3, p. 555-72, 1995. 


\section{DETERMINANTES DA DISSEMINAÇÃO VOLUNTÁRIA DE INFORMAÇÕES FINANCEIRAS NA INTERNET \\ Wesley Mendes-da-Silva - Paulo Azevedo de Oliveira Magalhães Filho}

MENDES-DA-SILVA, W.; ALVES, L. A. L. Internet and Voluntary Disclosure: Firm Value Effect Evidence. ANNUAL INFORMATION TECHNOLOGY CONGRESS. $1^{\text {st }}, 2004$, São Paulo. Anais. São Paulo : EAESP/FGV, 2004.

MITCHELL, J.; CHIA, C.; LOH, A. Voluntary Disclosure of Segment Information: Further Australian Evidence. Accounting and Finance, v. 35, n. 2, p. 1-16, 1995.

PIRCHEGGER, B.; WAGENHOFER, A. Financial Information on the Internet: A Survey of the Homepages of Australian Companies. European Accounting Review, v. 8, n. 2, p. 383-95, 1999.

SAVIN, N. E.; WHITE, K. The Durbin-Watson Test for Serial Correlation with Extreme Small Samples or Many Regressions. Econometrica, v. 45, p. 1989-96, Nov. 1977.

SHEEHY, D. E.; TRITES, G. D. Canadian accountant's guide to the Internet. Toronto: Carswell, 1997.

SINGHVI, S.; DESAI, H. An Empirical analysis of the Quality of Corporate Financial Disclosure. The Accounting Review, 46, p. 129-38, 1971.

SNEE, R. D. Some Aspects of Nonorthogonal Data analysis, Part I. Developing Prediction Equations. Journal of Quality Technology, v. 5, p. 67-79, 1973.

WAYMIRE, G. Earnings Vohatility and Voluntary Management Forecast Disclosure. Journal of Accounting Research, v. 23, p. 268-95, 1985.

ZINGALES, L. In Search of New Foundations. Journal of Finance, v. 55, n. 4, p. 1623-53, 2000.

\section{Artigo recebido em 16.04.2004. Aprovado em 08.03.2005.}

\section{Wesley Mendes -da-Silva}

Professor da Faculdade Integrada do Recife e Coordenador do Laboratório de Finanças do Recife. Mestre em Administração pela Universidade Federal de Pernambuco.

Interesses de pesquisa nas áreas de finanças corporativas, behavioral finance e emerging markets.

Email: wesley@ fir.br

Endereço: Av. Eng. Abdias de Carvalho, 1678, Madalena, Recife - PE, 50720-635.

\section{Paulo Azevedo de Oliveira Magalhães Filho}

Professor pesquisador da Matriz Empreendedora do Recife. Mestre em Administração pela Universidade Federal de Pernambuco.

Interesses de pesquisa nas áreas de estratégia empresarial, comportamento organizacional e sistemas de informação.

Email : paulom@ fir.br

Endereço: Av. Eng. Abdias de Carvalho, 1678, Madalena, Recife - PE, 50720-635. 